\title{
A Substitute to the Quantum Theory by an Entropy Approach
}

\author{
Salama Abdelhady \\ Energy Engineering College, Aswan University, Aswan, Egypt
}

\begin{abstract}
:
Following an entropy approach that replaced time by entropy in Maxwell's wave equation and reviewing experimental results, it was possible to prove a unique nature of electrons as particles, a unique nature of light as waves and to end the duality confusion. Additionally, the postulate of energy quantization into quanta each of magnitude " $h v "$ is proved as a conflict to the energy's nature as electromagnetic waves. Such conclusions led to violating main postulates of the quantum theory. Similarly, Schrodinger's equation that was introduced as a foundation of quantum mechanics to protest the duality confusions looses its meaning as the electron has a unique nature. According to the introduced entropy approach and measurement results, the photoelectric effect is found as a thermoelectric effect whose efficiency may approach the efficiency of Carnot cycle. Such conclusion violates the analysis of Shockley and Queisser that depends on the quantum approach and predicted an experimentally broken efficiency limit. As a conclusion, it is found that the entropy principles and the introduced entropy approach are rather realistic in microscopic analysis of thermodynamic systems when compared to the reviewed quantum theory.
\end{abstract}

Keywords: Quantum theory, quantum mechanics, entropy approach, electromagnetic waves.

\section{Introduction}

Quantum theory was started as a new branch of theoretical physics to study interactions of matter and radiation. The theory depends mainly on a description of electric current as a flow of electrons and on Einstein's explanation of photoelectric effect as collisions between radiation and electrons (Dirac, 1958). In his experiment, Felming found that the flow of electric charges has the velocity of light (Felming, 1911). Such measurement contradicts the assumed nature of electric current as a flow of electrons whose velocity is limited to the drift velocity of electrons of the order of few millimeters per second (Halliday, 2013). Similarly, Tesla succeeded in his famous experiment to transfer electric charges of extremely high potential through air of high electric resistance, (Tesla, 1904). Such experimental results show the nature of electric current as a flow of waves and it is not a flow of electrons.

Mackey found entropy as a unique function of time (Mackey, 1992). So, the time in Maxwell's wave equations was replaced by entropy to construct an energy frame of reference for thermodynamic analysis of the flow of electromagnetic waves. Such frame was a key to show that the electric current can be represented by a flow of electromagnetic waves that have an electric potential (Abdelhady, 2010a). Such conclusion assures the previously mentioned experimental results and deletes the 
confusions of the duality property of electrons (Abdelhady, 2011b). However, the duality property of electrons was considered as a central pivot of quantum theory or the one to which all other quantum mysteries can ultimately be violated (Liboff, 2002). Such result sustains the suspects of many scientists who considered quantum mechanics

In the following sections of this article, it will be discussed a plausible explanation of the photovoltaic effect that depends on the introduced entropy approach. It will be reviewed also the quantum approach and theory which is considered according to many scientists as an incomplete science that needs a deeper explanation while they claim they are not quite sure what it means (Nikolic, 2007). It will be firstly discussed the violation of the postulate of quantization of energy in energy-quanta of magnitude " $h v$ ". Then it will be discussed in the third section the robust proofs of the nature of electric current as a flow of electromagnetic waves. In the fourth section, thermodynamic analysis of the photoelectric effect that depends on the introduced entropy approach and experimental measurements will be discussed. Such analysis finds a plausible explanation of the recent advances in photovoltaic cells that have higher efficiencies than that defined by Shockley and Queisser limit (). Then, it will be compared the entropy approach to statistical thermodynamics as defined by Boltzmann to that defined by Schrodinger which depends on quantum theory. However, the introduced entropy approach was applied in a previous researches to developing the energy analysis of fiber optics, (Abdelhady, 2012b), to propose a universal system of units (Abdelhady, 2010b), and it is introduced here as a tool to improve the energy analysis of solar cells.

\section{Nature of Energy: Is it Quanta or waves?}

Transmission of energy is accomplished in general, by electromagnetic waves which are mathematically described by the Maxwell's wave equations (Halliday, 2013). Mackey found entropy as a unique function of time (Mackey, 1992). So, time in the Maxwell's equation may be substituted by entropy to represent the flow of electromagnetic waves into an energy frame of reference as seen in Figure 1 where the time axis is replaced by entropy axis. So, Maxwell's equation is written as follows (Abdelhady, 2010a):

$$
\begin{aligned}
& \left(\nabla^{2}-\frac{1}{c^{2}} \frac{\partial^{2}}{\partial s^{2}}\right) E=0 \\
& \left(\nabla^{2}-\frac{1}{c^{2}} \frac{\partial^{2}}{\partial s^{2}}\right) H=0
\end{aligned}
$$

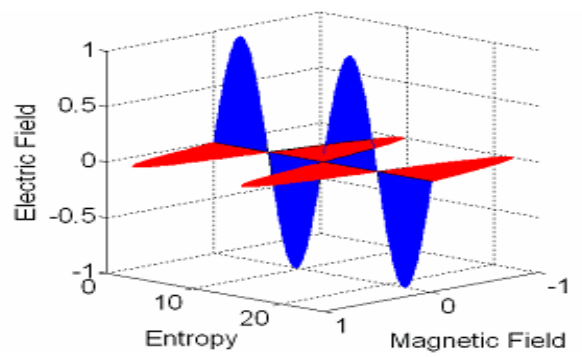


According to the found similarity between electric, magnetic, and thermal energies, it was possible to define all of them as electromagnetic waves and to express their fluxes as the product of the characterizing potential times the corresponding entropy flow (Abdelhady, 2010)

$$
\begin{aligned}
& \delta Q_{r e v}=T d S \\
& \delta Q_{e}=E d S_{e} \\
& \delta \Phi_{m a g}=H d S_{m a g}
\end{aligned}
$$

According to Equation (4), the integral of the left side " $\int_{1}^{2} T d \dot{S}$ " expresses the rate of heat transfer to a system during a process and is represented into a T-s property diagram by the area under the process line (Yunus, 2010). By similarity, Equations 5 and 6 led to define the red area in the E-s plane, in Figure 1, as area representing the flowing electric energy to the system and the blue area in the H-s plane in the same figure as the area representing the flowing magnetic energy. Denoting the sum of the imparted electric and magnetic energies per wave, as represented by the red and blue areas in Figure 1 by the symbol " $\tilde{h}$," then according to Equations 5 and 6 , " $\tilde{h} "$ is determined by the following equation:

$\tilde{h}=\int_{0}^{2 \pi}\left(\left|E d S_{e}\right|+\left|H d S_{\text {mag. }}\right|\right)$ Joule/wave

So the incident solar energy per unit area "I" can be expressed by the following equation:

$I=\tilde{h} \cdot n_{w} \cdot v \quad \mathrm{~W} / \mathrm{m}^{2}$

In this equation " $n_{w}$," is the number of incident waves per square meter, and " $v$ " is the frequency of the incident waves. As the incident energy is in the form of electromagnetic waves that transfers the energy into pulses of each of time $1 / v$ seconds, such pulse can be expressed in terms of the energy per wave as follows:

$\check{h}=\tilde{h} \cdot n_{w} \mathrm{~J} /$ pulse. $\mathrm{m}^{2}$

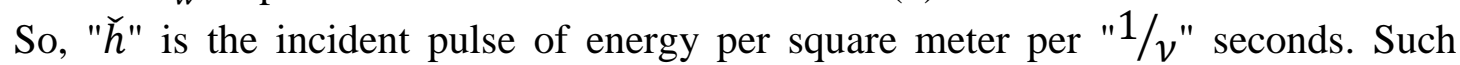
amount of energy can be estimated for each color of solar radiation to determine its impact on the output electric energy.

However, as an average value of solar irradiance, the figure $1000 \mathrm{~W} / \mathrm{m}^{2 "}$ is considered as a suitable value for most solar collectors or modules and is denoted in literature as "1 Sun" (Kurtz, 2009). Accordingly, it is possible to find the average value of the pulse of solar radiation by substituting the solar irradiance $I=1000 \mathrm{~W} / \mathrm{m}^{2}$ and the peak solar frequency $v=550.10^{12} / \mathrm{s}$ into equation (9) as follows:

$\breve{h}=\tilde{h} \cdot n_{w}=\frac{I_{1} \text { sun }}{v_{\text {solar }}}=1.82 * 10^{-12} \mathrm{~J} /$ pulse on $1 \mathrm{~m}^{2}$

This figure represents the value of one energy pulse that is sent from the sun as waves on one square meter in a time of $1 / v$ seconds. Accordingly, the incident solar radiation can be estimated in general according to the following equation:

$I=1.82 * 10^{-12}$ r.v $\mathrm{W} / \mathrm{m}^{2}$

In Equation 10, " $r$ " is the number of suns or the concentration ratio, and $v$ is the frequency of the incident radiation. According to Equation 11, the flow of 
electromagnetic waves through photovoltaic cells should be considered as a

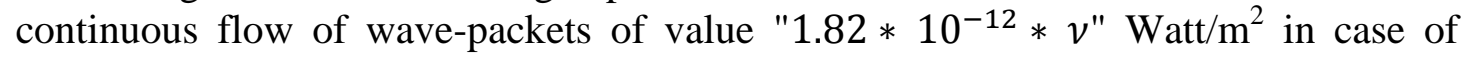
1 sun radiation.

However; quantum theory is based on the assumption of flow of energy in photons or quanta of energy each of magnitude " $h v$ " Joules. Such assumption contradicts the previous explanation of energy flow as electromagnetic waves according to the following:

1. If the energy transmission is described by the Maxwell's wave equations, then its frequency " $v$ " determines the rate of flow of such energy. Accordingly, the magnitude of its intermittent quantity in joules should not involve the frequency as a rate related parameter.

2. Considering the energy as a flow of electromagnetic waves that transfer packets or pulses of electric and magnetic energies whose sum is $\tilde{h}$ Joule per wave in a flow-time of $1 / v$ seconds contradict its flow into energy quanta whose magnitude has no relation to such pulses and its flow-time is unknown and is considered ineffective.

3. Considering the energy is flowing into waves of frequency" $v$," the units of Planck's constant " $h$ " as "J.s" hasn't any physical meaning in determination the value of its intermittent quantity or quanta.

Accordingly, the assumption that the energy flows into quanta of magnitude " $h v$ " contradicts the nature of energy flow as electromagnetic waves, contradicts the $\pi$ theorem of dimensional homogeneity, and has no physical meaning. The plausible description of such quantization should follow its nature as a wave, i.e. to be described as wave pulses of the magnitude $\tilde{h}$ in Joules per wave or $\breve{h}$ Joules per pulse. The value of each pulse in case of solar radiation, as calculated by Equation 10, equals $1.82 * 10^{-12} \mathrm{~J} /$ pulse on $1 \mathrm{~m}^{2}$ which is completely different from the quantum of undetermined time and a value of " $h v . "$

\section{Nature of Electric Current: Is it flow of electrons or waves?}

Figure 2 shows the graphical representation of the defined flow of electric energy as a flow of electromagnetic waves that have an electric potential (Abdelhady, 2010a). Felming experiments that measured the flow of electric current through conductor by the velocity of light contradicts the definition of electric current as electrons that flow by a drift velocity in the order of millimeters per second (Felming, 2009). Tesla's discovery of wireless power transmission of electric energy and the represents an experimental proof of the introduced definition (Abdelhady, 2013). So, the previous definition of electric current as a flow of electrons, while the electric current is a flow of electromagnetic waves, dresses the electron a wave's nature and causes the confusion duality that created a need to the quantum theory and mechanics (Abdelhady, 2011b). 


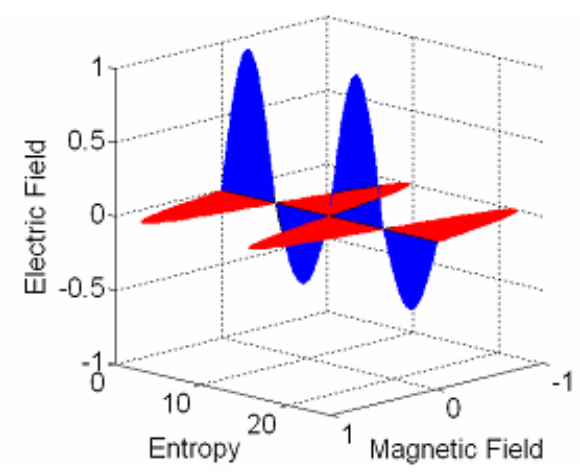

Figure 2 Graphical representation of a positive electric charge

Reviewing the experimental measurement of Planck's constant by a photoelectric cell, results of measurements are represented into a linear relation between the frequency of the incident waves and the opposing potential that stopped the current flow, Fig.4. At zero flow of current; the electrical potential of the reflected waves or charges is balanced by a stopping voltage. So, the emitted current should be defined as waves that have a potential that is balanced by such stopping voltage and it not just a flow of charges of the value "e" that haven't any or an unknown potential. The slope of the measured line in Figure 3 was defined according to the following equation (Hackworth, 2000):

$\theta=\frac{h c}{e}$

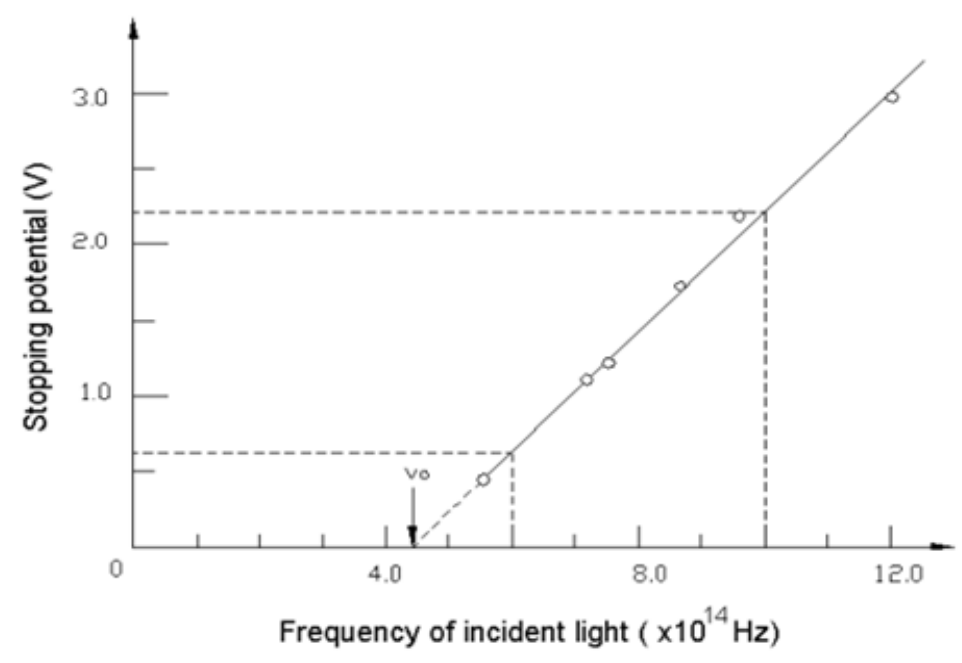

Figure 3 Plot of the stopping potential vs. frequency of incident light for photocell of Sodium Cathode (Hackworth, 2000)

If the flow of current is stopped by an opposing potential, it indicates the absence of flow of any electric current or charge. Hence, inserting a quantity of an electric charge to determine the slope of the measured line according to Equation 12 is meaningless. According to Figure 4, the value of the stopping potential, which is equal to the potential of the reflected wave or current, depends directly on the frequency of the incident waves. However, frequency of the incident radiation is function of the source 
temperature of the incident radiation according to the following Wien's law of radiation:

$\lambda_{\text {peak }}=\frac{0.0029}{T} m=\frac{c_{\text {light }}(\mathrm{m} / \mathrm{s})}{v_{\text {peak }}} m$

Substituting the speed of light, $c_{\text {light }}=300.10^{6} \mathrm{~m} / \mathrm{sec}$, we get the following relation between the source temperature and the frequency of the incident radiation:

$v=5.879 .10^{10} \mathrm{~T} \mathrm{~Hz}$

Hence, the measurement results define a relation between the thermal potential of the incident radiation and the induced potential of the reflected current. If the frequency in the ordinate of Figure 4 is replaced by temperature, according to Equation 13, then the relation between the thermal potential of the incident electromagnetic waves " $T_{S}$ " and the induced electric potential of the reflected waves or current " $V_{z}$ " can be expressed by the following equation:

$V_{z}=\theta \cdot T_{s} \quad$ Volt

" $\theta$ " is the value of the slope of the line which can be estimated according to the measurement data in Figure 4 as follows:

$\theta=40 \frac{\mu \text { Volt }}{\text { Kelvin }}$

Results of similar measurements of Planck's constant for two different metals of the photocell's plate show the same value of the " $\theta$ " that means it is independent on the metal's kind (Ducharme, 1999). However, this value of the induced potential difference per Kelvin in the photocells is of the same order of magnitude as the measured induced potentials, or electromotive force, in thermocouples as ChromelAlumel, Iron-Constantan, and Copper-Constantan which are found in the range of 42 $\mu$ Volt to $55 \mu$ Volt/ K. (Figiliola, 2009). According to such similarity of measured values, the photoelectric effect can be explained as a thermoelectric effect. So, the explanation of the photoelectric effect as a process of collisions between photons and electrons, as defined by Einstein, is violated (Liboff, 2002). The proof of violating Einstein's explanation also depends on the principles of conservation of momentum as it is impossible for a mass-less photon to eject an electron whose momentum is more than ten millions the momentum of such photon (Abdelhady, 2011a). The threshold frequency, as marked in Figure 3, can be plausibly explained as the minimum frequency or temperature of the incident radiation that can gain a sufficient potential to overcome the resistance against the current flow from the collector to the charge receiver.

\section{The Photovoltaic Effect: Is it a thermoelectric effect?}

According to classical quantum literature, the process of converting sun's radiation into electricity is called the photovoltaic effect and it is created when radiation from the sun hits a photovoltaic cell. These cells are made up of two layers of semiconducting material, typically silicon, which are called $\mathrm{P}$ and $\mathrm{N}$ layers (Sze, 1981) $\&($ Nelson, 2009). The boundary between these $\mathrm{P}$ and $\mathrm{N}$ layers is called as a PN junction and acts as a diode that allows electrons to move from $\mathrm{N}$ to $\mathrm{P}$, but not from $\mathrm{P}$ to $\mathrm{N}$. The classical explanation postulates when light with sufficient energy hits the cell, they cause electrons to move from the $\mathrm{N}$ layer to the P layer causing excess 
electrons in the N-layer and a shortage in the $\mathrm{P}$ layer that results in voltage drop across the junction and allows the flow of electric current. According to the principles of conservation of momentum, the momentum of the light waves, or the claimed photons if they exist, cannot move electrons whose momentum is ten millions the claimed momentum of such photons, to move. It is possible to pass such quantum explanation in case of silicon cells that are supposed to have electrons on one side and holes on the other side due to doping by different materials. But it is not logical to accept such explanation in case of thin-film solar cells which are formed by deposition of two different semiconductors in absence of imaginable free electrons and holes or in case of multi-junction solar cells which lacks logically these electrons and holes in each layer (Lukue, 2006).

Reviewing the performance of photovoltaic cells as shown in Figure 4, the gained potential by the incident radiation during its flow across the junction is seen to be independent on the intensity of solar radiation but it is function of the frequency or the thermal potential of the incident radiation. According to the measurement data; the open circuit voltage, $\mathrm{V}_{\mathrm{oc}}$, has the value of 0.55 Volts where the temperature difference between the sun's temperature and the junction's temperature is $5600 \mathrm{~K}$. Hence, the potential rise per Kelvin is in the order $60-70 \mu \mathrm{V} / \mathrm{K}$. Such values are also near to the value of the generated e.m.f. in most of thermocouple junctions as ChromelAlumel, Iron-Constantan, and Copper-Constantan which are in the range of $42 \mu \mathrm{V} / \mathrm{K}$ to $55 \mu \mathrm{V} / \mathrm{K}$ (Figiliola, 2009). The equality of these ranges indicates the incident solar radiation on the photovoltaic cells gains a potential difference when it crosses the PN junction of photovoltaic cell by the same effect that induces an electromotive force into heat that crosses the thermocouple junction by the Seebeck thermoelectric effect (HKUST, 2009). So, the photoelectric effect and the photovoltaic effect are not more than thermoelectric effects.

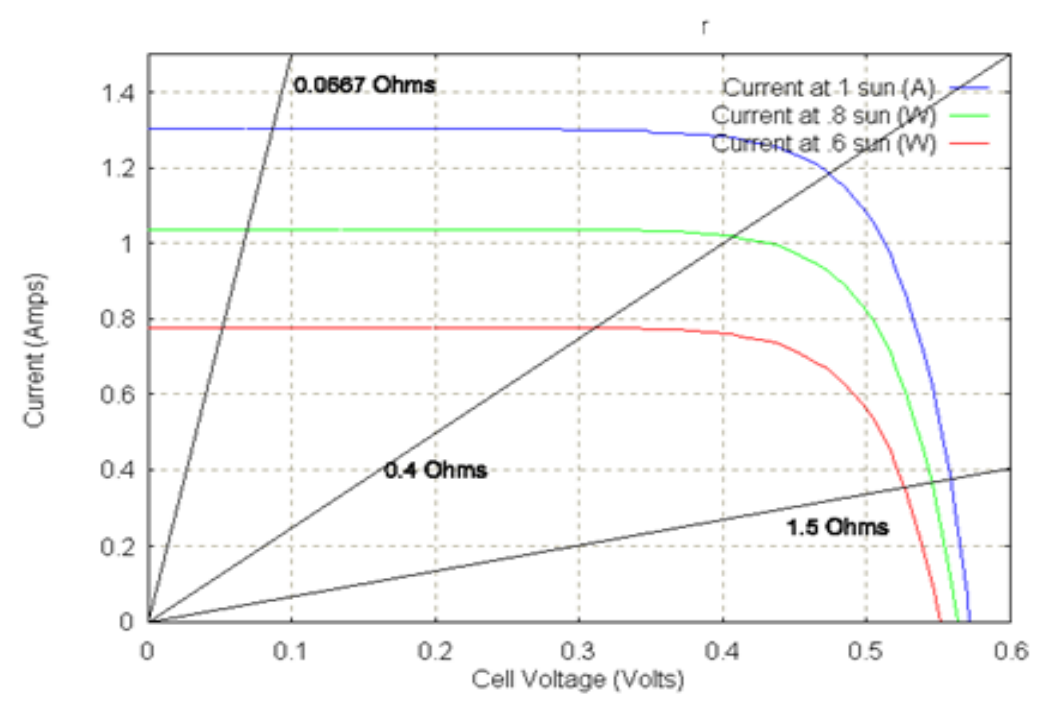

Figure 4. Basic photovoltaic-cell characteristics

The similarity of the processes of photovoltaic junctions and thermocouple junctions as voltage sources is identified by an identical technique which is applied in both of 
them to increasing or magnifying their voltage outputs by using multiple-junction thermocouples, or thermopiles, multi-junction photovoltaic cells, and multicouple thermoelectric generator, Figures 5, 6 and 7. In case of multi-junction solar cells, each junction gives a potential difference to the output current, as indicated in Figure 6. So, the resultant potential will be the sum of such potentials and the output power will be determined by product of the current time the total potential that increase the efficiency of the cell and reduce its costs (Stuart, 2011). Similarly, the total potential of the multiple-junction thermocouple, or thermobile, has a magnification scale determined by the number of junctions (Figiliola, 2009).

In case of thermoelectric power generators that is used in direct conversion of the incident heat energy into electricity by Seebeck effect the same principles of using multicouple or multi-junctions is applied to duplicate the generated power, Figure 6 (Weiling, 2004). For a typical Bi2 Te3 thermoelectric generator, the generated e.m.f is in the order of $220 \mu \mathrm{V} / \mathrm{K}$ for each couple or $55 \mu \mathrm{V} / \mathrm{K}$ from each junction (Weiling, 2004). Such value is in the same order of magnitude of the thermocouple junctions and photovoltaic cells. In addition, the characteristics of this thermoelectric generator as shown in Figure 7 are similar to the characteristics of photovoltaic cells as shown in Figure 7.

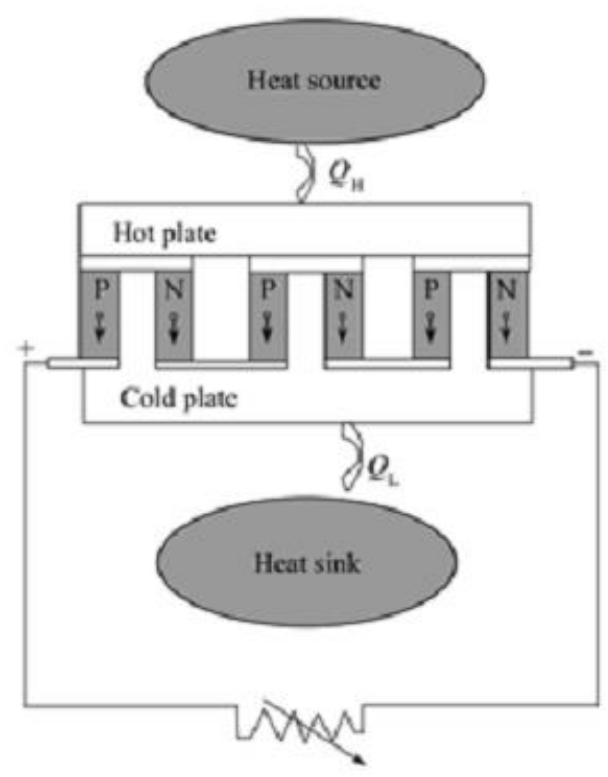

Figure 6. Schematic diagram of multicouple thermoelectric generator (Weiling, 2004) 


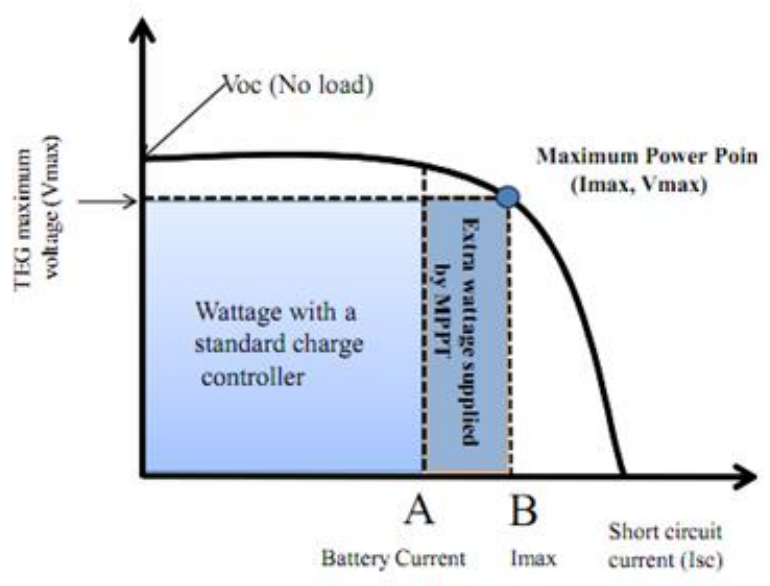

Figure 7. Characteristics of thermoelectric generator (Weiling, 2004)

The found similarity in the value of potential increase per degree, the similarity of applied multi-junction techniques and the similarity of the characteristics of photocells and the thermoelectric generator, as shown in Figure 5 and 7 represent the sufficient proof that the photovoltaic effect is a thermoelectric effect.

Applying an energy equation that embraces a flow of electric energy on a control volume that surrounds a photovoltaic cell as shown in Figure, the following equation is obtained (Abdelhady, 2010):

$$
T_{S} \dot{S}_{t h}=E \dot{S}_{e}+T_{0} \dot{S}_{t h}
$$

The term on the L.H.S. of Equation 11 represents the rate of flow of thermal radiation into the junction expressed as the temperature of the input radiation " $T_{S}$ " times the rate of flow of thermal entropy, " $\dot{S}_{t h}$ ". The first term in the R.H.S. of the equation is the outflow rate of electric energy expressed as the product of an induced potential $E$ times the rate of flow of electric entropy " $\dot{S}_{e} . "$

Considering the photovoltaic cell as a thermoelectric generator, the second law of thermodynamics states that it is impossible to have an engine whose efficiency is higher than that of the ideal Carnot cycle. According to the exergy principles, the minimum loss of energy or unavailable energy in such cell, or engine is equal the junction temperature $T_{0}$ times the rate of flow of the thermal entropy " $\dot{S}_{t h}$." By rearranging Equation 17, it can be found the following relation:

$$
\begin{aligned}
& E \dot{S}_{e}=\dot{S}_{t h}\left(T_{S}-T_{0}\right) \\
& \frac{\dot{S}_{t h}}{\dot{S}_{e}}=\frac{E}{\left(T_{S}-T_{0}\right)}
\end{aligned}
$$

Defining the efficiency of the solar cell as follows:

$$
\eta=\frac{\text { output electric energy }}{\text { input thermal energy }}=\frac{E \dot{S}_{e}}{T_{S} \dot{S}_{t h}}
$$


Substituting the output electric energy according to Equation 18 into Equation 20:

$\eta==\frac{\left(T_{S}-T_{0}\right) \dot{S}_{t h}}{T_{S} \dot{S}_{t h}}=\frac{\left(T_{S}-T_{0}\right)}{T_{S}}=1-\frac{T_{0}}{T_{S}}$

According to the followed entropy approach, the efficiency of the photovoltaic cell as a thermoelectric generator has a limiting efficiency that equals to that of Carnot cycle efficiency. Substituting the temperatures of the sun and the junction at normal conditions in Equation 21:

$\eta=1-\frac{T_{0}}{T_{S}}=1-\frac{302}{6100}=0.95$

According to the found results, it is possible to reach an efficiency of $95 \%$. However, to get such efficiency or the stated output in Equation 18, the ratios of thermal to electric entropy flows should be equal to the ratio of the source temperature and the induced potential in the junction according to Equation 19. According to the measurement data found in many measurements, Figure 9, satisfaction of such condition depends on the thermoelectric properties of the junction materials that allow the ratio of flow of such rates of entropy through the junction and through the load, $\frac{\dot{s}_{t h}}{\dot{s}_{e}}$, to be equal to the matched potential across the load to the temperature difference between the sun and the junction, $\frac{E}{\left(T_{S}-T_{0}\right)}$. So, adaptation of such condition is a resonant point at which the cell reaches its maximum power point indicated in Figure 7. So, the industry of photovoltaic cells should search the selection of the junction materials or the multi-junction cells to increase the efficiency of photovoltaic cells.

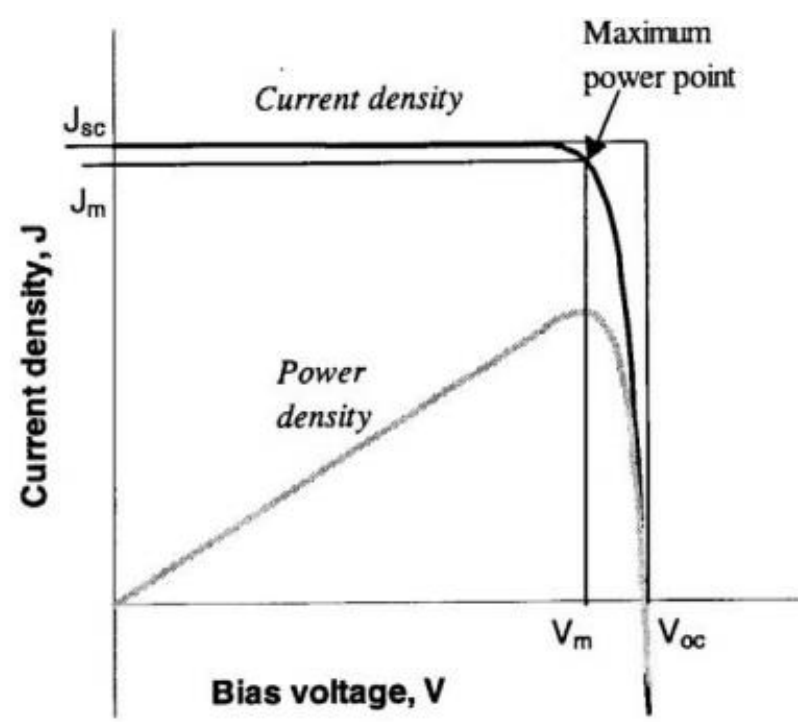

Figure 8. Output Power of photovoltaic cell reaches maximum power at a maximum power point determined by load adjustment (Nelson, 2009).

Reviewing the analysis of performance of PV cells as developed by Shockley and Queisser to find the efficiency limit of a single junction PV cell, they built their 
insight purely on an analysis which is deeply rooted to the second law of thermodynamics. Their analysis considers also the Carnot cycle efficiency, as found in Equation 21 according to the previous entropy approach, as the efficiency limit of converting the incident thermal radiation into electric energy (Shockley, 1961). So, the analysis of Shockley and Queisser sustains the introduced entropy approach that considers the photovoltaic cell has similar characteristics of a thermoelectric generator. However, Shockley and Queisser involved in their thermodynamic analysis a further quantum-constraint. They considered a recombination mechanism of the imagined hole-electron pairs that depends on a quantum approach. Such approach is restricted to the definition of electric current as a flow of electrons and Einstein's explanation of photoelectric effects as interactions between radiation and particles. According to literature and available measurements, Shockley and Queisser limit was broken by modern designs of photovoltaic cells (Kurtz, 2009). Accordingly, Shockley and Queisser succeeded in introducing the second law analysis as a tool to investigate the conversion of thermal energy to electric energy as a thermoelectric effect but their approach was deviated by introducing the quantum interactions into such analysis (Shockley, 1961). So, the measured photovoltaic efficiency that exceeds the Shockley and Queisser limit represents a proof of failure of the quantum theory in finding the correct explanation of the photovoltaic effect.

\section{A Comparison between Boltzmann and Schrodinger's Approaches to Microscopic or Statistical Thermodynamics}

According to Boltzmann's principles (Barkai, 2004), the entropy change between two states of the thermodynamic system is related to the logarithm of the probability $\mathrm{W}$ of a spontaneous energy transition between the two states by the formula

$S=k \ln W$

So, entropy as a thermodynamic quantity links, according to Equation (23), the macroscopic properties of a system to its microscopic states and determines what sorts of transformations a system will undergo. Such equation was derived from a macroscopic analysis of an ideal gas during an expansion process. The basic rule is that thermal systems will tend to states of higher entropy. So the entropy difference between two states of a system gives us information on the tendency of the system to move between the states. Indeed the "tendency" can be given a quite precise measure as a probability. If we know the entropy difference between two states of a system, we know the probability that the system will spontaneously move between those two states.

According to quantum theory, electrons are assumed to have wavelike properties; and a particular wave equation. Schrodinger equation governs how these waves behave according to imaginary positions of electrons in free space as a tool to microscopic description of systems (Walter, 1992). According to the introduced entropy approach and the entropy principles, as defined by Boltzmann, we are not obliged to take such Schrodinger's imaginary solutions and quantum explanation as an approach to the microscopic analysis of real systems as the electrons are found only as particles. 


\section{Conclusions:}

According to the introduced entropy approach and results of experimental measurements, the photovoltaic effect is found as a thermoelectric effect whose efficiency may approach the efficiency of Carnot cycle. Such approach proves that the quantum analysis of such effect that depends on the definition of current as flow of electrons and on assuming the behavior of energy quantum of magnitude " $h v "$ as particles failed in predicting the measured efficiency limit of the photovoltaic cells. It is also found that such entropy approach is rather realistic in the microscopic analysis of thermodynamic systems in comparison to the quantum approach.

\section{References}

Abdelhady, S. (2010a). "A Fundamental Equation of Thermodynamics that Embraces Electrical and Magnetic Potentials," Journal of Electromagnetic Analysis \& Applications, 2: pp. 162-166, http://dx.doi.org./10.4236/jemaa.2010.23023/

Abdelhady, S. (2010b) "An Approach to a Universal System of Units," Journal of Electromagnetic Analysis \& Applications, , 2: pp. 549-556, September, 2010. http://dx.doi.org./10.4236/jemaa.2010.2907/

Abdelhady, S. (2010c). "Comments Concerning Measurements and Equations in Electromagnetism ," Journal of Electromagnetic Analysis \& Applications, 5: pp. 677 678. http://dx.doi.org./10.4236/jemaa.2010.212089

Abdelhady, S. (2011a). "Comments on Einstein's Explanation of Electrons, Photons, and the Photo-Electric Effect," Applied Physics Research, 3 (2), pp. 230-240. http://dx.doi.org./5539/apr.v3n2p230/

Abdelhady, S. (2011b). "An Entropy-Approach to the Duality Property," Journal of Electromagnetic Analysis \& Applications, 2 (3): pp.220- 227

Abdelhady, S. (2012) "A Thermodynamic Analysis of Energy Flow in Optical Fiber Communication Systems," Applied Physics Research, Vol. 4, No.3, pp. 22-29, August 2012. http://dx.doi.org./10.5539/apr.v4np22

Abdelhady, S. (2013). “An Entropy Approach to Tesla's discovery of Wireless Power Transmission," Journal of Electromagnetic Analysis \& Applications, 5: pp. 157-161, April, 2013. http://dx.doi.org./10.4236/jemaa.2013.54025/

Barkai, E. (2004). A Linear Boltzmann Equation Approach, Journal of Static Physics, Nos. 516.

Cotal, H., Fetzer, C., Boisvert, J., Kinsey, G., King, R., Hebert, P., Yoon, H., and Karam, N. (2008). III-V Multi-junction Solar Cells for Concentrating Photovoltaics, Review, Energy \& Environment Science, Doi: 101039/b809257e

Dirac, P. A. M. (1958). The Principles of Quantum Mechanics (4th ed.). Oxford University Press. 
Ducharme, S. (1999) "Measuring Planck's Constant with LEDs", Retrieved form: http://physics.unl.edu/directory/ducharme/ducharme.html

Ellerman, D. (2011).A Very Common Fallacy in Quantum Mechanics, Retrieved from: http://arxiv.org/abs/1112.4522

Fleming, J. A. (1911). Propagation of electric currents in telephone \& telegraph conductors. New York: Van Nostrand.

Figliola, R.E., Beasley, D. F. (2009)."Theory and Design for Mechanical Measurement," (Fifth Edition), Lohn Wiley \& Suns Inc.

Hackworth, M. (2000)"Measuring Planck's Constant", Retrieved from, http://www2.cose.isu.edu/ hackmart/planck's.PDF

HKUST, Department of Physics. (2009). "Photoelectric Effect," Retrieved from: http://hkpho.phys.ust.hk/Training/Experiments/127Lab1_PhotoelectricEffect.pdf

Halliday, D., Resnick, R., \& Walker, J. (2013). Fundamentals of Physics, (10 th edition), New York: John Wiley \&Sons

Kurtz, S. (2009)." Technical Report, Opportunities and Challenges for Development of a Mature concentrating Photovoltaic Power Industry, NREL/TP-520-43208.

Liboff, R. (2002). Introductory Quantum Mechanics (4th ed.). Addison Wesley. ISBN $\underline{0-8053-8714-5 .}$.

Lukue, A., Hegedus, S. (2003). Photovoltaic Science and Engineering, John Wiley \& Sons

Mackey, M. C. (1992). Time's Arrow: The Origins of Thermodynamic Behavior. Berlin Heidelberg New York: Springer. ISBN 3-540-94093-6. OCLC 28585247.

Nelson, J. (2009). The Physics of Solar Cells, Imperial College Press

Nikolic, H. (2007). Quantum Mechanics: Myths and Facts. Foundations of Physics, 37(11): p.1563-611

Shockley, W., Queisser, H.J. (1961). Detailed Balance Limit of Efficiency of P-N Junction Solar Cells, Journal of Applied Physics 32, pp.510-519

Stuart, R. W., Martin, A. G. (2011), Annual Report, ARC Photovoltaic Center of Excellence.

Styer, D.F. (1996), Common Misconceptions Regarding Quantum Mechanic, American Journal of Physics, 64, pp. 31-34

Sze, S. M. (1981). "Physics of Semiconductor Devices", John Wiley \& Sons, New York. 
Walter, J. M. (1992). Schrödinger: Life and Thought. Cambridge University Press. ISBN $\underline{0-521-43767-9 .}$.

Weiling, L, Shantung, T. (2004). "Recent developments of thermoelectric power generation," Chinese Science Bulletin, , Vol. 49, No. 12. Pp.1212-1219

Yunus, C., Michael, A.B., (2010)." Thermodynamics: An Engineering Approach,” McGraw-Hill, Boston: Science Engineering,

Mackey, M. C. (1992). Time's Arrow: The Origins of Thermodynamic Behavior. Berlin Heidelberg New York: Springer. ISBN 3-540-94093-6. OCLC 28585247. 